\title{
Système d'information géographique et de visualisation de l'aléa inondation Application aux crues de la Marne
}

\author{
par C. Brachet, ISL bureau d'ingénieurs-conseils, \\ P. Bolo, Aqualis
}

\section{I — APPLICATION DES SIG À L'ÉTUDE DES CRUES DE LA MARNE}

L'utilisation répandue des Systèmes d'Information Géographique (SIG), la disponibilité des sources de données et la progression technique ouvrent de multiples applications concernant le thème hydraulique. Nous présentons ici un logiciel utilisé en amont et en aval des codes de calcul hydrauliques de propagation des crues. Facilitant l'intégration des données et améliorant la présentation des résultats, il permet une analyse rapide et rigoureuse ainsi qu'une meilleure représentation cartographique des risques.

Cet outil est actuellement utilisé sur la Marne dans le cadre d'une analyse des écoulements. Celle-ci vise notamment à appréhender l'effet des inondations fréquentes à exceptionnelles ainsi qu'à aider à la définition de scénarios d'aménagement. Ceux-ci doivent permettre de réduire les dommages induits pour les événements de moyenne occurrence. Le schéma d'aménagement tient compte de l'ensemble des fonctionnements du milieu constitué par la plaine alluviale, ceux liés à l'expansion des crues étant prépondérants dans le contexte de la Marne.

\section{LA PLAINE ALLUVIALE}

\section{- 2.1. L'échelle de travail}

Les mesures préventives du risque d'inondation en France sont orientées par les Schémas Directeurs d'Aménagement et de Gestion des Eaux [1] et s'appuient sur les Plans de Prévention des Risques (PPR). Elles visent à proscrire tout aménagement susceptible de subir des dommages dans les zones inondables, ainsi qu'à conserver les zones naturelles d'expansion des crues.
Le territoire du bassin de la Marne couvre un linéaire important de vallée. Outre l'élaboration des PPR à des échelles cadastrales, une vision plus globale s'impose. Une modélisation hydraulique à maille lâche sur la Marne moyenne a ainsi été associée à des investigations plus précises sur les agglomérations situées en zones sensibles.

L'échelle de travail est le 1/50 000e, cette précision étant suffisante pour répondre à l'objectif global du schéma d'aménagement qui est la programmation des actions. On souligne que cette échelle n'est pas opérationnelle pour la réalisation des PPR.

\subsection{Géomorphologie des vallées de la Marne}

La limite de l'extension des crues exceptionnelles est déterminée par l'emprise des formations alluvionnaires récentes [2]. Cette zone constitue la limite latérale du système faisant l'objet des modélisations hydrauliques et cartographiques. Les alluvions recouvrent les plaines sur la Marne moyenne ainsi qu'entre les Ornains. On note les zones de large extension alluvionnaire du Perthois ou encore du Marais de St-Gond.

Le bassin versant de la Marne occupe une superficie de $12700 \mathrm{~km}^{2}$, soit $30 \%$ de la superficie du bassin de la Seine en amont de Paris. Les alluvions modernes se retrouvent sur une superficie d'environ $900 \mathrm{~km}^{2}$, le tiers constituant le lit majeur de la Marne moyenne.

L'approche géomorphologique qualitative constitue un préliminaire à l'interprétation quantitative, celle-ci s'appuyant sur la modélisation numérique des crues [3]. Elle aide à la construction topologique des éléments du modèle, à la valorisation des données topographiques et bathymétriques, au choix des rugosités et à la compréhension de la dynamique fluviale. La morphologie du lit ordinaire permet d'autre part d'apprécier son débit à plein bord par tronçon.

Geographical Information Systems (GIS) are used in flood studies. Combined with an hydraulic modeling of the french Mame river, impacts of various river-works may be appreciated, ranging from river restoration to dam building. Geomorphological approach at a rated scale is preliminar to flood quantification. Hydraulic and cartographic models come afterwards. Geographical confrontation of those results to land cover gives the risk for the different schemes. 


\subsection{Les fonctions} de la plaine alluviale

Les fonctionnalités des plaines alluviales relevant de la gestion de l'eau sont : le transfert de l'eau d'amont vers l'aval, l'expansion, le stockage et le laminage des crues, l'alimentation et le drainage de la nappe alluviale, le contrôle de la qualité des eaux ; les autres fonctions concernent le transfert de matière solide, l'habitat pour la flore et la faune, le maintien de la biodiversité, la transformation des nutriments, le support d'activités et la production de paysages [4].

L'échelle de travail et la limitation de la zone d'étude à la plaine alluviale situent la perception au niveau du paysage intermédiaire de la vallée.

La rivière est appréhendée dans sa globalité à travers l'étude du lit mineur et de la zone d'expansion des crues. Les autres niveaux de perception numérique des rivières encadrent la précédente : il s'agit de la codification du réseau hydrographique et de l'exutoire du bassin versant, ce dernier niveau s'appliquant à l'hydrologie [5].

\section{III — MODÉLISATIONS DES CRUES}

\section{- 3.1. Les outils numériques}

L'étude des zones inondables nécessite une réflexion multi-critère qui intègre des informations sur la géomorphologie, la climatologie, l'hydrologie, la vulnérabilité des différents terrains submersibles, l'économie... La prise en compte de ces différentes sources d'informations - qui peuvent concerner d'importantes surfaces - nécessite une approche synthétique. Les SIG présentent l'avantage de pouvoir combiner des informations diverses sous forme de représentation spatiale. Ces outils permettent l'élaboration de cartes numériques des zones inondables dont la mise à jour devient simple et rapide.

L'exploitation des SIG offre ainsi des moyens performants pour l'étude des crues. L'utilisation de SIG rasteur (travaillant sous forme matricielle) permet d'intégrer facilement différents types d'informations surfaciques. Le mode rasteur offre la possibilité de réaliser de nombreuses combinaisons (arithmétiques, logiques, conditionnelles) entre les couches d'information.

La modélisation hydraulique est réalisée en modes filaires et quasi-bidimensionnels (ce dernier mode s'appliquant aux zones de stockage sans inertie) suivant les tronçons. Les simulations sont effectuées en régime transitoire afin de restituer les durées de submersion et l'effet de laminage du lit majeur.

\subsection{La modélisation du terrain}

A la sectorisation morphologique de la vallée et du lit de la rivière se superposent les limites administratives. Sur ces limites, deux natures du terrain sont modélisées : le modelé de surface et l'occupation du sol. Le premier thème est approché sous la forme d'un modèle numérique de terrain, le deuxième sous la forme d'une occupation des sols arrêtée à une époque précise.

Le modèle numérique de terrain peut être scindé en une surface générale du terrain et en une micro-topographie. Celle-ci comprend les éléments de drainage et le sursol, celui-ci étant entre autres constitué des remblais linéaires (routes, talus). Une attention particulière est apportée à la modélisation du lit mineur de la rivière et des ouvrages hydrauliquement significatifs.

Les sources du modèle numérique de terrain peuvent être de diverses natures : levers au sol, restitution photogrammétrique, extraction manuelle (digitalisation) ou automatique (intelligence artificielle) de courbes de niveau d'un fond de plan existant. C'est une digitalisation complétée par des profils en travers du lit majeur qui a été utilisée sur la Marne moyenne. Les dimensions et les profils en long des émissaires principaux et des ouvrages sont précisés par des levers au sol.

\section{- 3.3. La modélisation hydraulique}

Le SIG permet une intégration aisée et rigoureuse des données, tant topographiques qu'hydrologiques, en prétraitement des calculs hydrauliques. L'utilisation du modèle numérique de terrain et d'un support photographique, combinée à une étude préliminaire géomorphologique, permet d'établir une topologie fine du modèle, qu'il s'agisse de modélisation filaire ou quasi-bidimensionnelle.

Concernant l'hydrologie, la prise en compte des apports intermédiaires et des interactions avec les nappes est facilitée par l'utilisation du SIG. Les attributs caractéristiques des 


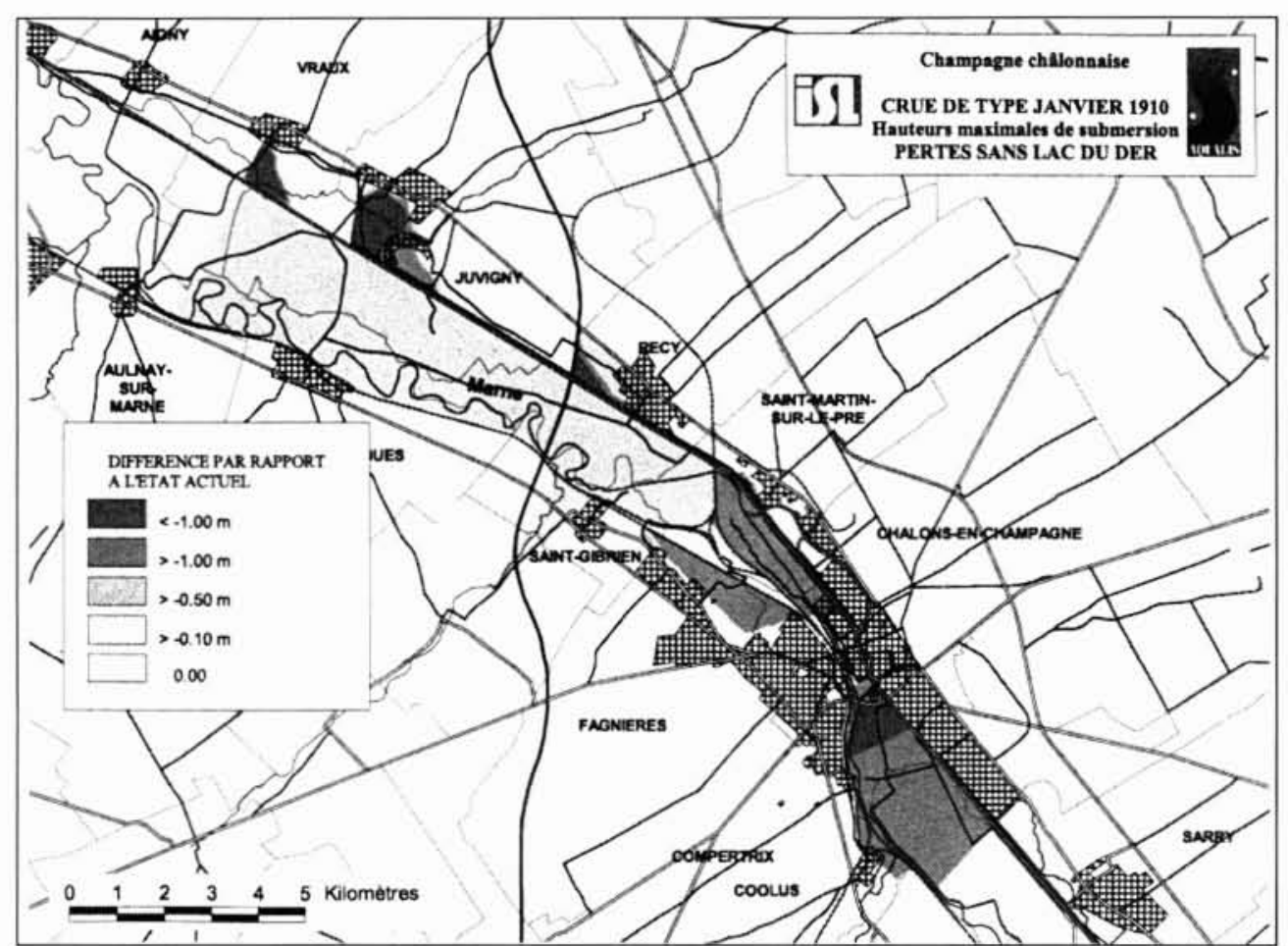

2. Différences des hauteurs pour une crue de type 1910 entre l'état actuel et sans le lac du Der.

\subsection{Conclusion}

L'ensemble des données utilisées dans l'étude des crues ainsi que des résultats produits peuvent l'être sous forme numérique. Les analyses statistiques, la diffusion et la mise à jour s'en trouvent facilitées [7]. La démarche adoptée sur la Marne a d'autre part l'avantage de permettre la cohérence (préconisée dans le SDAGE [1]) des actions de prévention et de protection contre les inondations à l'échelle du bassin versant. Les études réalisées s'appuient sur le Système Hydraulique d'Information et de Visualisation de l'Aléa (ou SHIVA) développé par ISL et AQUALIS.

L'étude du fonctionnement du contrôle des crues révèle une certaine homogénéité sur l'ensem-

bassins versants sont attribués aux exutoires correspondants. Les crues simulées sont celles de 1910 et de 1983, celle-ci servant également au réglage du modèle [6].

\section{- 3.4. L'appréciation du risque}

Trois termes de l'aléa permettent l'évaluation du risque lié à une crue : la hauteur d'eau (fig 1), la durée de submersion et la vitesse du courant. Différents degrés d'appréciation sont distingués suivant la méthodologie d'approche.

La simple approche géomorphologique permet d'apprécier l'aléa hauteur d'eau, sans intégrer précisément l'action anthropique ni la dynamique fluviale. L'hydrologie permet d'appréhender globalement les durées de submersion, en distinguant les phases montante et descendante de la crue. L'écrêtement peut alors être mis en évidence par secteurs et éventuellement croisé aux largeurs d'inondation.

La modélisation hydraulique permet, une fois le réglage validé, de simuler des projets d'aménagement (fig 2). Elle renseigne précisément sur l'aléa dû aux hauteurs d'eau et aux durées de submersion. L'appréciation des vitesses nécessite une interprétation tenant compte de la bande de méandrement et de la micro-topographie.

Les dégâts aux habitations et aux infrastructures sont conditionnés par les hauteurs et les vitesses. Concernant les zones de cultures et de prairies, les dommages dépendent essentiellement des durées de submersion pour une date fixée. La dynamique de la crue peut être représentée par une animation présentant l'évolution des surfaces inondées au cours du temps.

La confrontation de l'aléa et de l'occupation du sol permet l'obtention des dommages économiques. Les gains entre l'état actuel et l'état projeté suivant différents scénarios sont mis en regard des coûts d'aménagement. Une cartographie thématique de la qualité de l'habitat, des débits de débordement, des perturbations hydrauliques et des zones sensibles est également réalisée. ble du linéaire des vallées de la Marne. Les problématiques restent proches dans la Région parisienne et sur le reste du bassin, des situations comparables concernant les pôles urbains localisés à l'amont d'une zone de transition située entre Meaux et Noisy-le-Grand. Quel que soit le secteur, l'essentiel de la lutte contre un événement de type 1910 reste une question de prévision et d'anticipation des crues ; le tout se trouve facilité par la culture du risque. Ce mode de lutte peut cependant s'avérer insuffisant dans l'auréole englobant les têtes de bassin.

Sur la Marne rurale, nombre de syndicats cuvrent pour la protection contre les inondations. Leur expérience et leur connaissance du cours d'eau pourrait être mise à profit, en collaboration avec les services techniques, dans le cadre des plans de gestion des risques.

\section{BIBLIOGRAPHIE}

[1] Bassin Seine-Normandie - Schéma Directeur d'Aménagement et de Gestion des Eaux, approuvé le 20/09/96.

[2] De la carte géologique aux applications : approche d'une délimitation des zones inondables par la cartographie des dépôts alluvionnaires récents, Langevin\&Gresselin, 1996.

[3] Cartographie des Zones inondables - Approche hydrogéomorphologique, Masson\&Garry\&Ballais, 1996.

[4] Les Fonctions des Zones humides - Synthèse bibliographique, Fustec\&Frochot, 1996.

[5] Utilisation conjointe de SIG et modèles hydrologiques pour les études d'incidence sur les cours d'eau, Mokadem\&Dautrebande, 1991.

[6] Atlas des Zones inondées en Avril 1983, AESN.

[7] Les SIG pour gérer le risque d'inondation. Bonviller\&Fouchier. 1997. 